\title{
SPINAL WITHDRAWAL REFLEXES IN THE HUMAN LOWER LIMBS
}

BY

\section{K. E. HAGBARTH}

\section{From the Department of Clinical Neurophysiology, the University Hospital, Upsala, Sweden}

In his original description of the nociceptive flexion reflex in vertibrate animals Sherrington (1910) declares that even though the reflex is most readily elicited from the foot, it has a receptive field which includes "the skin of the whole limb as far up as the groin in front, the perineum medially, and the ischial region behind". He states that noxious stimuli within this field cause not only a contraction of the flexors of hip and knee and the dorsiflexors of foot and digits, but also a relaxation of antagonistic extensors and plantar flexors. In addition, he points out that even though the ensuing movement is always a flexion of the limb "there comes to be some accentuation of movement at this joint or that according as the skin-point stimulated lies in this limb-region or that" (the phenomenon of "local sign").

It was recognized quite early, however, that ipsilateral extension reflexes could also be elicited from the skin or the cutaneous nerves. The "extensor thrust" represents an example of such a reflex, and it was also noted that modification of the stimulus applied to sensory nerves sometimes changed the motor response obtained from a flexion to an extension reflex (Sherrington and Sowton, 1911; Brown, 1912; Brown and Sherrington, 1912). These authors' final conclusion was that in vertibrate animals there exist ipsilateral extension reflexes of cutaneous origin; they are often concealed by the dominant flexion reflex but they can be uncovered by varying the site and nature of the stimulus. In an attempt to make a further analysis of these spinal extension responses, Hagbarth (1952) mapped the receptive skin fields for various flexor and extensor muscles in the cat's hind leg and found that local extension reflexes could be elicited from certain skin areas which were mainly localized over the extensor muscles themselves.

Formerly many clinicians believed that extension reflexes could not appear after a complete transection of the spinal cord in human beings, but observations of Kuhn (1951) and other workers have shown that this is not so. An extensive study of the cutaneous reflexes obtainable from the lower limbs of patients with spastic paraplegia was carried out by Marshall@ (1954) who demonstrated that ipsilateral extension reflexes could be elicited from various skin areas onthe leg, thigh, and perineum and that the nature of the skin stimulus often determined whether flexion or extension ensued. He found no clear evidence that ${ }^{\circ}$ extension reflexes were more easily elicited from skin overlying extensor muscles than from flexor surfaces.

The nociceptive cutaneous reflexes in the lowerw limbs of man have also been investigated with ap $\omega$ electromyographic technique (Kugelberg, 194⿳亠丷厂犬; Hoffmann, Schenck, and Tönnies, 1948; Dodt argd Koehler, 1950; Pedersen, 1954) but so far systematic analysis of the receptive fields for individual muscles has been performed, and thus it is still unknown if the local extension reflexes seen in the cat exist also in man. It seemed that such ân analysis of spinal skin reflexes in healthy subjecc $\vec{\otimes}$ might help to clarify the mechanisms responsible. for "abnormal" reflex patterns characteristic of $O$ upper motor neuron disease.

\section{Methods}

Most experiments were performed on the author himself, others on medical students. The subject was sitting, lying, or standing in different positions while noxious electric stimuli were systematically applied to different skin areas in one of the lower limbs. The stimuli were obtained by letting an electric current? (square wave pulses at 500 to $1,000 / \mathrm{sec}$.) pass through the sharp tips of a small bipolar needle electrode (interpolar distance 2 to $3 \mathrm{~mm}$.) held in contact with the skin. A current of 5 to $10 \mathrm{~mA}$., lasting 10 to $30 \mathrm{~m}$.sec. was usually sufficient to cause an intense burning sensation from the skin and a withdrawal reaction of the extremity.

Muscular activity was recorded with bipolar needle electrodes and after adequate amplification the potentials $O$ were displayed on the beam of a cathode-ray oscilloscope, the sweep of which was synchronized with the electrical skin stimulus; in order to visualize not only excitatory but also inhibitory effects of the stimulus a background $\mathrm{N}$ of slight "voluntary" contraction was usually maintained in the muscles recorded. Three to five stimuli in succession were regularly applied to each skin area of the limb 
and the ensuing reflexes, as displayed on the oscilloscope, were superimposed on photographic paper.

\section{Results}

A series of preliminary experiments showed how various muscles in the extremity react to a noxious stimulus applied most distally on the plantar surface of the hallux. After a latency varying from about 60 to $80 \mathrm{~m} . \mathrm{sec}$. a reflex discharge appeared in some of the muscles while others were inhibited. The reflexes were regularly followed by "silent periods" and the inhibitions by secondary discharges. Occasionally the cyclic activity persisted for a longer time and involved several alternating periods of excitation and inhibition, a phenomenon which has earlier been described both in connexion with the flexion reflex (Hoffmann, et al., 1948; Schenck and Koehler, 1949) and the abdominal skin reflex in man (Kugelberg and Hagbarth, 1958). When attention was focused on the initial effect of the stimulus, however, it turned out that the following muscles responded with a reflex discharge: The tensor fasciae femoris, the sartorius, the rectus femoris, the tibialis anticus, the peroneus longus, and the extensor brevis digitorum, while the follow- ing were inhibited: The gluteus maximus, the vastus medialis, the vastus lateralis, the gastrocnemius, the soleus, and the flexor brevis digitorum. The responses in the semimembranosus and the semitendiknosus were somewhat variable but usually the former was inhibited while a reflex appeared in the latter.

The muscles listed agree very well with those enumerated by Sherrington (1910) in his description of the muscles participating in the flexion reflex of vertebrate animals. Moreover, the latencies of the responses are short enough to indicate that these reflexes in man are also mediated by spinal reflex arcs (cf. Kugelberg, 1948; Pedersen, 1954). It is consequently safe to conclude that a noxious stimulus to the plantar surface of the hallux in man evokes a reflex which closely resembles the flexion reflex of Sherrington. As will be shown, however, other reflex patterns appear when the skin stimulus is applied to more proximal parts of the limb.

Fig. 1 shows how the response in the gluteus maximus changes as the skin stimulus is systematically moved from one skin area to another along the ventral and dorsal aspect of the entire limb. This muscle was inhibited from most skin areas but it
Fig. 1. - Reflex responses in the gluteus maximus evoked by noxious skin stimuli within various regions of the limb. The shadow indicates the localization of the muscle investigated and the radiating lines indicate from which skin areas the various responses were elicited. Black bars in the beginning of each record show the duration of the stimulus. The lower middle record shows the control background activity in the muscle. Three to five sweeps are superimposed in each record. Time, 100 c.p.s. Cal. 0.5 $\mathrm{mV}$.

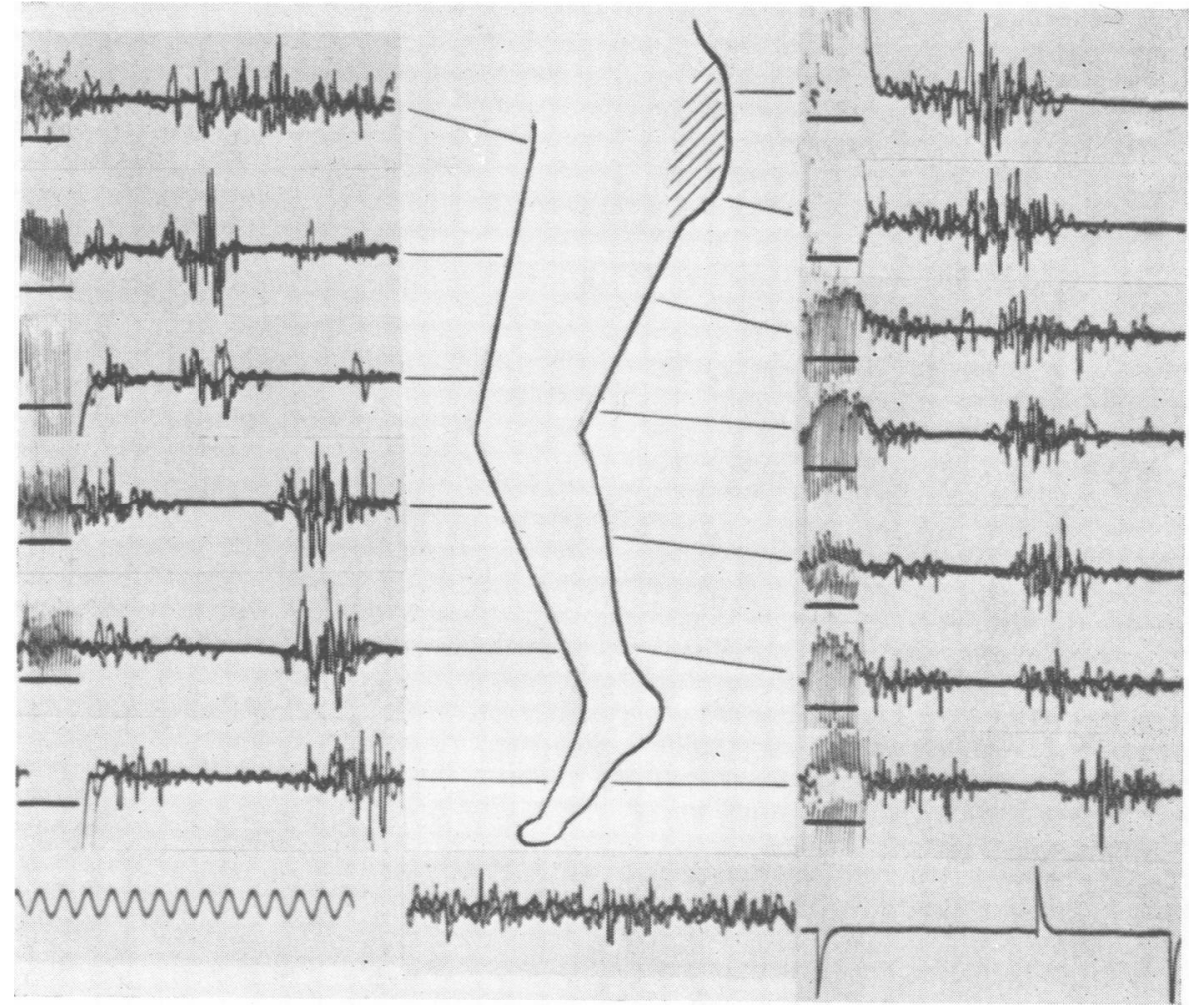




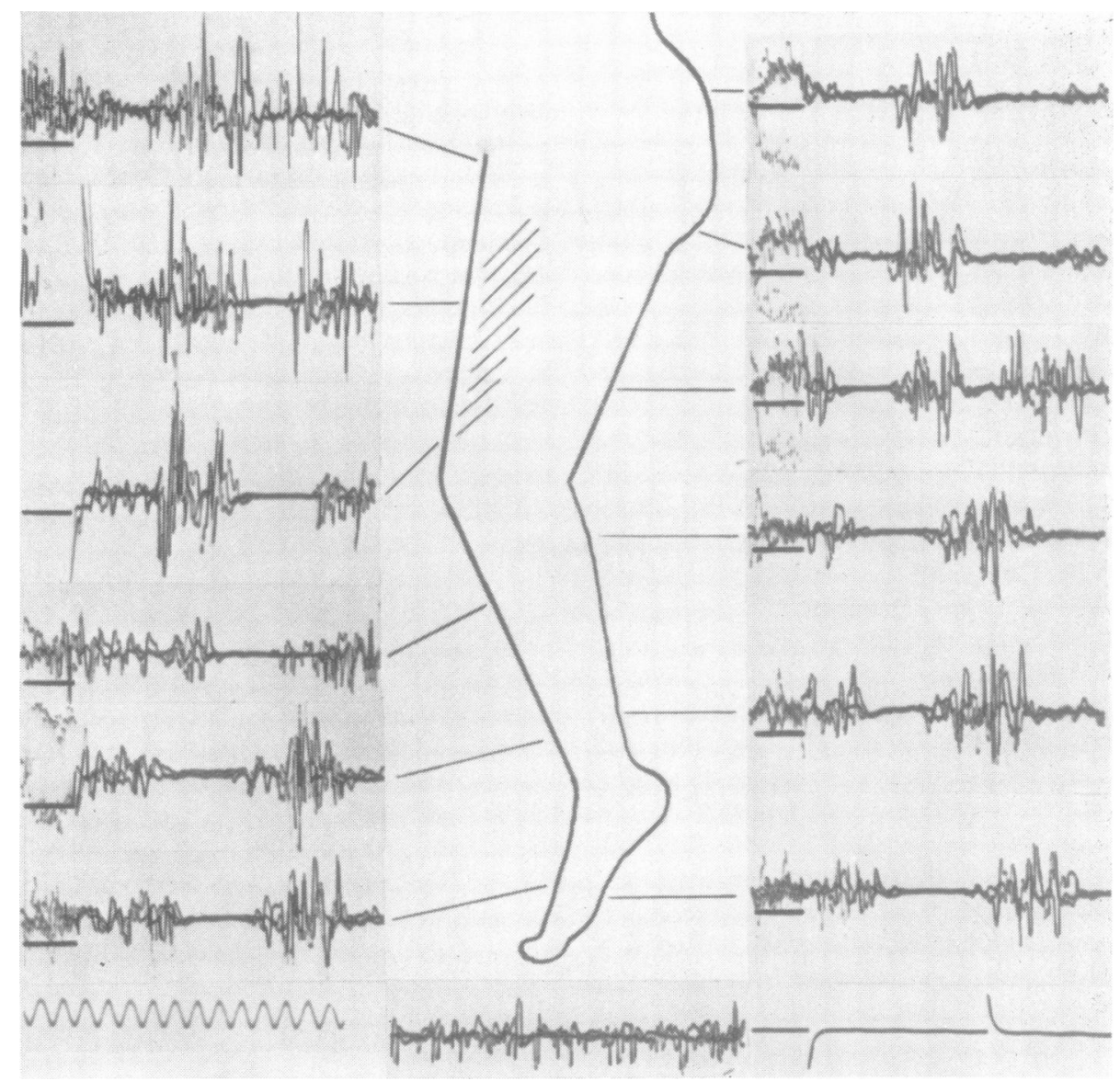

FIG. 2.-Responses in the vastus medialis to skin stimuli of various localizations. For further description see legend to Fig. 1. Time, 100 c.p.s. Cal. $0.5 \mathrm{mV}$.

regularly responded with a brisk initial reflex discharge to skin stimuli in the gluteal region. It was not possible to define any exact boundaries between the excitatory and inhibitory skin fields. Individual variations were observed, but usually the reversal of the response appeared gradually as the stimulus was moved in a proximal direction on the dorsal aspect of the thigh.

As shown in Fig. 1, the duration of the initial inhibitory phase tended to decrease as the stimulus was shifted further proximally. In particular the responses elicited from the ventral part of the thigh were characterized by a short inhibitory phase followed by a rapid secondary discharge. The most probable explanation of this phenomenon seems to be that the more proximal the stimulus, the less dispersed the sensory signals entering the reflex centre. As a consequence the immediate motor response is better synchronized and the ensuing cyclic changes are likely to reach higher frequencies, be these due to intraspinal mechanisms or external circuits.

The next muscle investigated was the knee extensor, the vastus medialis (Fig. 2). It was inhibited from most skin areas of the limb, including the gluteal region, but usually initial discharges appeared when stimulating the ventral part of the thigh. The superposition technique proved to be of great value in these experiments where it was important to distinguish between primary excitatory effects and rebound discharges following a short initial inhibition. Occasionally a stimulus to the ventral part of the thigh caused a brief initial inhibition in the vastus medialis, but then a small shift of the electrode position was all that was needed to recover the primary excitatory effect.

A closer study of Figs. 1 and 2 reveals how the latency of the responses gradually decreases as the 
FIG. 3.-Responses in the gastrocnemius (medial head) to skin stimuli within various limb regions. For further description see legend to Fig. 1. Time, 100 c.p.s. Cal. $0.5 \mathrm{mV}$.
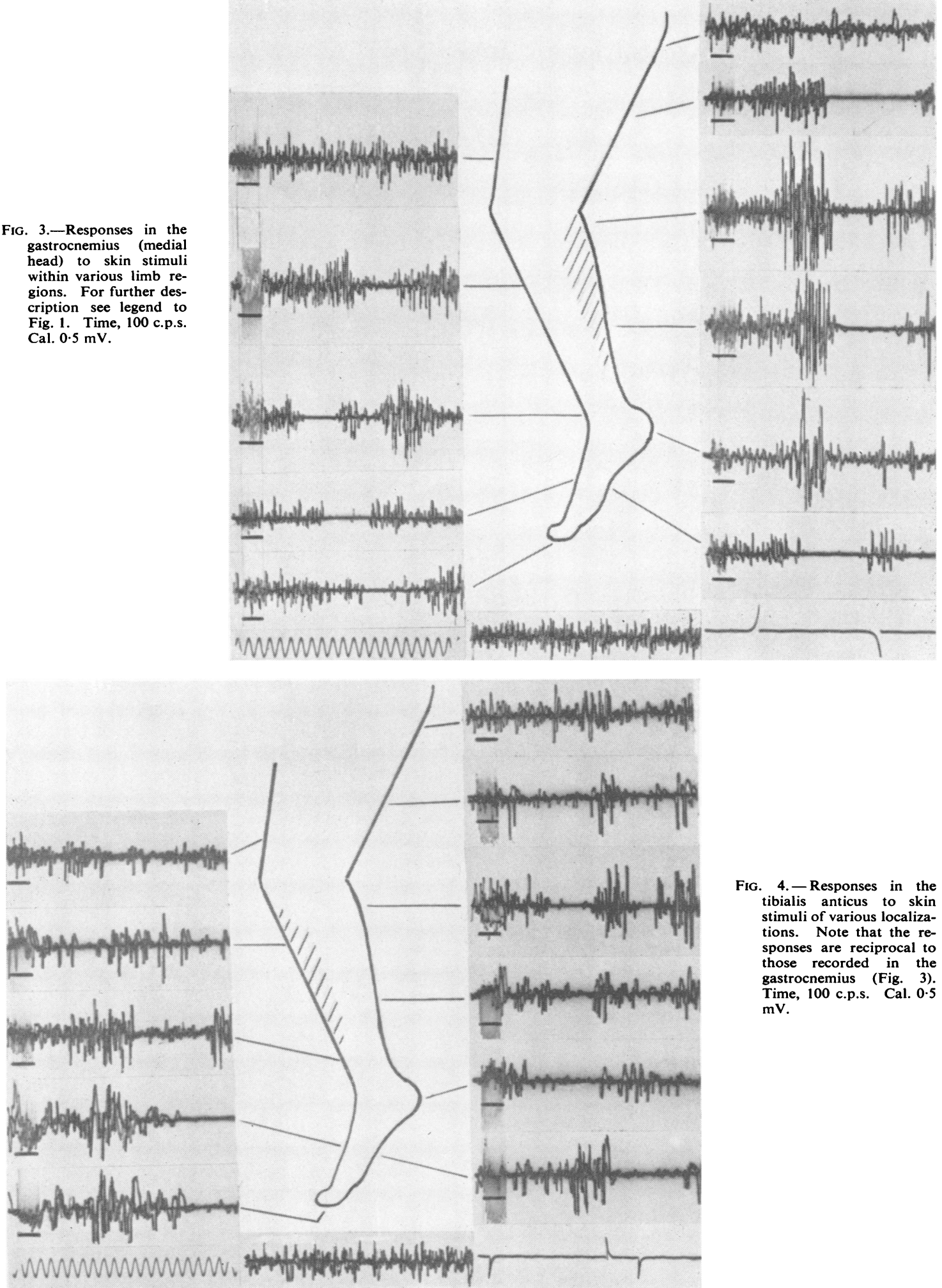

FIG. 4.-Responses in the tibialis anticus to skin stimuli of various localizations. Note that the responses are reciprocal to those recorded in the gastrocnemius (Fig. 3). Time, 100 c.p.s. Cal. 0.5 $\mathrm{mV}$. 
stimulus is moved upwards on the limb. The latency of the initial inhibition decreases from about 65 to $35-40 \mathrm{~m} . \mathrm{sec}$. as the stimulus is moved from the foot to the perineal or ischial region, a distance which in this subject was about $1 \mathrm{~m}$. Assuming that variations of intraspinal conduction time can be neglected, this would correspond to a sensory conduction velocity of 33 to $40 \mathrm{~m} . \mathrm{sec}$., a rate which indicates that even larger sensory fibres than those of the delta group are involved (cf. Kugelberg, 1948; Pedersen, 1954). It should be added that the stimulus intensity in the present experiments was kept as constant as possible in order to minimize latency changes due to variations in the strength of the stimulus.

Occasionally it appeared as if the primary discharges, elicited from the gluteal region and the ventral part of the thigh respectively, had a somewhat longer latency than the inhibitory responses evoked from neighbouring skin areas. This impression, however, was mainly obtained in those instances when the discharges had a more gradual onset than the inhibitory effects. Minor differences were therefore hard to evaluate and on the whole the two types of responses had similar latencies.

The gastrocnemius and the soleus, the plantar flexors of the foot, behave, according to Sherrington, as extensor muscles inasmuch as they are usually inhibited by ipsilateral noxious skin stimuli. In the present study these calf muscles were found to react to the skin stimuli in the following way. Stimuli to the dorsal side of the limb, from heel to thigh, caused an initial reflex discharge while stimuli to most parts of the foot caused inhibition. As a rule, small initial discharges could be elicited also from certain areas on the ventral side of the leg while stimuli on the ventral side of the thigh usually were ineffective (Fig. 3).

The tibialis anticus, the prime antagonist to the calf muscles, responded to the skin stimuli in a reciprocal way. It was consequently inhibited from those skin areas which had an excitatory effect on the gastrocnemius and the soleus (Fig. 4).

As a rule, the responses of the various muscles appeared most distinctly when displayed against a "voluntary" background activity of moderate strength. The gastrocnemius, which is a doublejoint muscle, was "voluntarily" activated either as a plantar-flexor of the foot or as a flexor of the knee. The mode of activation, however, had no appreciable influence on the reflex patterns described. These were also approximately the same whether the subject was standing on the leg, sitting, or lying in different positions.

From the clinical point of view, the plantar- and dorsi-flexors of the toes attract a special interest as they are the main participants in the normal plantar $\stackrel{\bar{c}}{\mathrm{c}}$ and Babinski responses. The way in which these $Z$ muscles react to ipsilateral noxious skin stimuli forms the main theme in a separate and independent study on by Eklund, Grimby, and Kugelberg (1959).

\section{Discussion}

It seems very likely that the reflexes described in the present paper are of cutaneous origin. The shape of the stimulating electrodes and the parameters of the electrical current were especially $\stackrel{5}{\stackrel{5}{*}}$ chosen to give a superficial burning sensation from $\frac{}{2}$ the skin and it was repeatedly checked that the 흠 stimuli did not cause any radiating pain, or any $\frac{\infty}{\widetilde{D}}$ indirect muscle contractions, even when the electrode $\propto$ was placed on purpose over some of the main motor $\%$ nerve trunks.

As already mentioned, it also seems safe to conclude that the reflexes are mediated by spinal reflex $\vec{\omega}$ arcs. The gradual decline of latency as the stimulus is moved proximally (shown for the inhibitory responses in Figs. 1 and 2) allows not only an approximate estimation of the conduction velocity $\mathrm{N}_{\mathrm{U}}$ of the sensory fibres mediating the flexion refles $\omega$ ( 33 to $40 \mathrm{~m} . \mathrm{sec}$.), it also indicates that the centrâ] delay must be relatively short. As shown in Fig. $\Phi_{9}^{N}$ the inhibitory responses in the gluteus maxims elicited from the perineal region had a latency of $\rightarrow$ 35 to $40 \mathrm{~m} . \mathrm{sec}$. and in this case it was estimated that the conduction distance to the spinal cord coupde hardly be less than $45 \mathrm{~cm}$. With a sensory conduction velocity of 33 to $40 \mathrm{~m} . \mathrm{sec}$. this means $\mathrm{a}$ peripheral sensory delay of about $13 \mathrm{~m} . \mathrm{sec}$. and. consequently 22 to $27 \mathrm{~m} . \mathrm{sec}$. are left for central delay and motor transmission. Considering that allowance should be made for a period of temporal summation, this delay can hardly permit participation of pathways higher than spinal in the basic $\frac{\mathbb{D}}{2}$ reflex arcs (cf. Dawson, 1947). Since the latencies $\Rightarrow$ are approximately the same for the inhibitory and $\frac{0}{3}$ excitatory effects on the extensor muscles, it must be assumed that the latter kind of responses also are mediated by spinal paths.

Fig. 5 summarizes the results by showing the distribution of the excitatory and inhibitory skin:areas for the muscles investigated. It must be emphasized that the boundaries between the different areas are often quite vague and also that individual 3 variations may exist which have not been encountered in the present study. Even so, it seems? justifiable to present this schematic drawing as a basis for further discussion.

The results agree fairly well with those obtained N in vertebrate cats inasmuch as the extensor muscles tend to be excited from skin areas mainly localized 


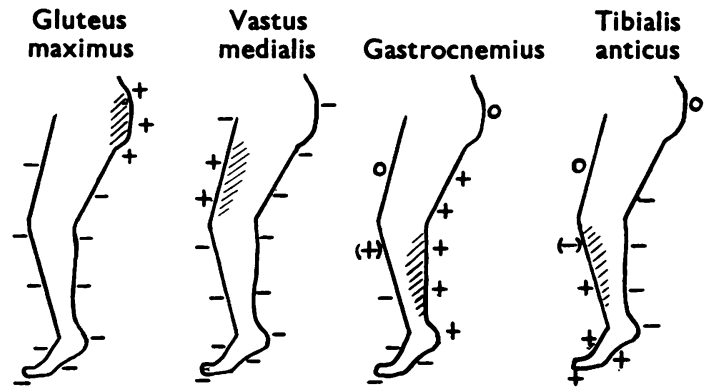

FIG. 5.-Diagrams showing the approximate extent of inhibitory and excitatory skin areas for the muscles investigated.

over the extensors themselves (Hagbarth, 1952). This generalization certainly does not hold in a strict anatomical sense but it serves the purpose of describing the main results in relatively few words.

There is little doubt that the main biological purpose of the ipsilateral nociceptive skin reflexes is to cause a rapid movement away from the offending object. To be effective, such withdrawal movements must be quite specific in regard to the localization of the skin stimulus and it is easy to realize that an extension movement often serves the protective purpose better than a flexion. The initial position of the limb, however, must also be considered when it comes to evaluating the withdrawal capacity of a motor pattern. The protective function of the local extension reflexes becomes apparent only if it is assumed that the subject at the time of the stimulus is walking or standing on the limb (see Fig. 5). When the calf muscles in this situation contract to a noxious stimulus on the dorsum of the leg, the lifting of the heel initiates a step which brings the limb away from the stimulus. It is also evident that extension of the knee tends to cause withdrawal from a stimulus on the distal ventral part of the thigh and that extension of the hip is the adequate withdrawal reaction to a stimulus in the gluteal region. It should again be pointed out, however, that the local extension reflexes were elicitable not only when the subject was standing on the leg, but also when he was sitting or lying in various positions.

It is by no means a novel observation that skin stimuli to the buttocks may evoke a reflex discharge in the gluteal muscles. Neurologists have long been familiar with the fact that a skin stroke in the ischial region tends to evoke a gluteal contraction and it is also known that a loss of this reflex may be indicative of pyramidal disease. The gluteal reflex is usually regarded as analogous to the abdominal skin reflexes which, no doubt, have certain properties in common with the local limb reflexes described in the present paper. It was recently shown (Kugelberg and Hagbarth, 1958) that the abdominal skin reflexes are part of an elaborate spinal defence mechanism which is so organized that independently of whether a noxious skin stimulus is applied to the front, side, or back of the trunk, it evokes a contraction in adjacent underlying muscles and a reciprocal inhibition of antagonists on the opposite side of the trunk. The various local cutaneous trunk reflexes, which Duensing (1952) described as a sign of extrapyramidal disease, may also serve to exemplify how skin stimuli tend to evoke reflex contractions in adjacent underlying muscles.

At present, experiments are being performed to find out how the local extension reflexes are affected in pyramidal and extrapyramidal disease. Preliminary results indicate that the reflexes are often weak or absent in patients with spastic paralysis while they may be quite vivid in athetosis. The number of patients investigated is still too small, however, to warrant any definite conclusions.

\section{Summary}

Spinal nociceptive skin reflexes in the human lower limbs have been analysed with an electromyographic technique. It is shown that the reflex patterns vary considerably according to the localization of the ipsilateral skin stimulus. Besides the patterns of the flexion reflex there are local extension reflexes which are mainly elicitable from skin areas covering extensor muscles. It is believed that these local extension reflexes serve a protective purpose in that they can cause an effective withdrawal of the heel, knee, and buttock, especially when the limb supports the body weight.

\section{REFERENCES}

Brown, T. G. (1912). Quart. J. exp. Physiol., 5, 237.

, and Sherrington, C. S. (1912). J. Physiol., (Lond.) 44, 125.

Dawson, G. D. (1947). J. Neurol. Neurosurg. Psychiat., 10, 137.

Dodt, E., and Koehler, B. (1950). Pfüg. Arch. ges. Physiol., 252, 362

Duensing, F. (1952). J. nerv. ment. Dis., 116, 973.

Eklund, K., Grimby, L., and Kugelberg, E. (1959). Acta physiol. scand., 47, 297

Hagbarth, K. E.'(1952). Acta physiol. scand., 26, suppl. 94.

Hoffmann, P., Schenck, E., and Tönnies, J. F. (1948). Pflüg. Arch. ges. Physiol., 250, 724.

Kugelberg, E. (1948). Brain, 71, 304.

, and Hagbarth, K. E. (1958). Ibid., 81, 290.

Kuhn, R. A. (1951). J. nerv. ment. Dis., 113, 301.

Marshall, J. (1954). Brain, 77, 290.

Pedersen, E. (1954). Acta psychiat. neurol. scand., Suppl. 88.

Schenck, E., and Koehler, B. (1949). Pflüg. Arch. ges. Physiol., 251, 504.

Sherrington, C. S. (1910). J. Physiol. (Lond.), 40, 28

-, and Sowton, S. C. M. (1911). Proc. roy. Soc., 83в, 435. 